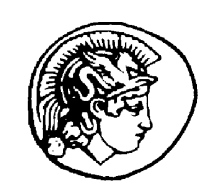

\title{
Assembly features in modeling and planning
}

\author{
Winfried van Holland ${ }^{1}$, Willem F. Bronsvoort* \\ Faculty of Information Technology and Systems, Delft University of Technology, Zuidplantsoen 4, 2628 BZ Delft, Netherlands \\ Received 16 March 1999; accepted 22 August 1999
}

\begin{abstract}
In recent years, features have been introduced in modeling and planning for manufacturing of parts. Such features combine geometric and functional information. Here it is shown that the feature concept is also useful in assembly modeling and planning. For modeling and planning of both single parts and assemblies, an integrated object-oriented product model is introduced. For specific assembly-related information, assembly features are used. Handling features contain information for handling components, connection features information on connections between components. A prototype modeling environment has been developed. The product model has been successfully verified within several analyses and planning modules, in particular stability analyses, grip planning, motion planning and assembly sequence planning. Altogether, feature-based product models for assembly can considerably help in both assembly modeling and planning, on the one hand by integrating single-part and assembly modeling, and on the other hand by integrating modeling and planning. (C) 2000 Elsevier Science Ltd. All rights reserved.
\end{abstract}

Keywords: Product modeling; Feature modeling; Assembly planning; Features; Assembly features

\section{Introduction}

In assembly modeling, a model is created of a product consisting of several components. Such a model contains, in particular, information about the mutual relations between the components. Most components consist of several smaller components, those that do not are called single parts. Due to the occurrence of single parts, assembly modeling cannot be separated from single-part modeling. The term product modeling is used to refer to both, and a product model therefore contains information about single parts, components and their relations.

Decisions made during product modeling, can have much impact on the complete life cycle of the product. A designer therefore has to take into account requirements from other disciplines involved in the life cycle of the product, e.g. marketing, manufacturing, assembly, service and disassembly. Ideally, several analyses should be automatically performed during modeling to check whether such requirements are met. For example, in the

\footnotetext{
* Corresponding author.

E-mail addresses: wvholland@baan.nl (W. van Holland), bronsvoort@cs.tudelft.nl (W.F. Bronsvoort).

${ }^{1}$ Current address: Baan Company NV, Baan Research and Development, P.O. Box 143, 3770 AC, Barneveld, The Netherlands.
}

context of assembly, it should be analyzed whether a product can be efficiently assembled with the available equipment. This concept is called $\mathrm{D}$ (esign) $\mathrm{F}$ (or) $\mathrm{X}$, where the $\mathrm{X}$ can be any life cycle phase, e.g. Manufacturing, Assembly or Service [1].

In assembly planning, plans are created that specify how the product can be assembled, given the product model. The difference between single-part manufacturing planning and assembly planning, is comparable to the difference between single-part modeling and assembly modeling. Product planning is therefore used to refer to both.

Both product modeling and product planning are highly dependent on well-defined product models. Examples of information stored in product models are the geometry of the product and the used material. Currently, there is hardly any integration between single-part models and assembly models, nor between product models used in modeling and planning. This results in several different product models for a product, at least one for every discipline involved in modeling and planning, and gives rise to severe problems, because of redundancy of data and loss of information due to conversions between models. A possible solution to this is the use of one integrated product model by all disciplines. Information can be stored by one discipline, and be used by other disciplines. 
In single-part modeling, there is now a shift from storing only geometry-oriented information towards more function-oriented information. This is done using features in feature-based models [2]. The functional information is very useful during modeling and planning of parts. Many computations can be done on features, instead of on pure geometry, which is much more effective.

In this paper, the feature-based concept will be applied to integrated modeling and planning of both single parts and assemblies. An object-oriented feature-based product model is described in Section 2. To represent specific assembly information in the product model, assembly features are used. These are defined and illustrated in Section 3. A prototype system has been implemented in which models can be created and, in addition, several analysis and planning modules can be activated directly from the modeling environment, thus creating an integrated modeling and planning system suitable for DFA. The prototype system is discussed in Section 4. The way the product model can be profitably used during assembly analysis and planning, in particular stability analysis, grip planning, motion planning and assembly sequence planning, is shown in Section 5. Some conclusions are presented in Section 6.

\section{Feature-based product model}

In this section, a feature-based product model is described suitable for modeling both single parts and assemblies. This idea was already mentioned by Shah and Rogers [3]. They considered a feature model for single parts as an 'assembly' of form features. Fig. 1(a) shows a simple part containing two form features, and a constraint to specify their relative position. Fig. 1(b) shows the same shape, but now containing two components, and a mating relation to specify their relative position. Both the single-part and the assembly model contain 'building blocks' and relations between them.

Although there exist similarities between single-part and assembly models, there are also differences, in building blocks and in relation types between the building blocks. Whereas in single-part modeling building blocks can be used that subtract volume, in assembly modeling only additive building blocks are used. To specify a product model for both, an object-oriented approach is used. The $\mathrm{OMT}^{2}$ class diagram notation is used for illustration purposes.

\subsection{Single-part model}

A single part can contain several building blocks of the same type, i.e. several instances of the same form feature.

\footnotetext{
${ }^{2}$ OMT stands for Object Modeling Technique as described by Rumbaugh et al. [4].
}

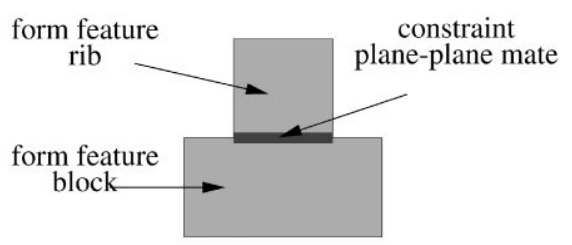

(a)

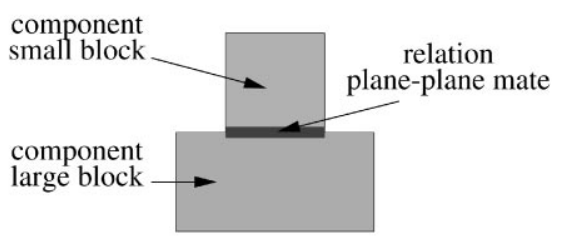

(b)

Fig. 1. Models for single parts and assemblies. (a) Single part model, containing two form features, and a constraint to specify their relative position; (b) assembly model of the same shape, but now containing two components, and a mating relation to specify their relative position.

Each type of form feature is represented by a GenericFormFeature class. Take, for example, a single part with multiple holes in it. Each hole is represented by a specific instance, called object in object orientation, of the Hole class. Every feature inherits from the base FormFeature class. This class contains a data structure in which the geometry of the feature can be described and operations, or methods, on these data structures with some specific functionality. Each derived feature class, e.g. the ThroughHole class, derives the data elements and operations on them from its base class, and makes some modifications on these data elements and operations to describe the geometry of that specific feature. Each instance of these classes, describes the exact shape, with specified attributes, e.g. a through hole with specified diameter attribute M8. Detailed descriptions of form feature classes can be found in, for example, Ovtcharova et al. [5], and an example is shown in Fig. 2.

A special class is the CompoundFormFeature class, where new form features can be created by taking combinations of other form features. In this way, the set of already known form features can be extended. Therefore we need an association relation between classes. In the OMT notation, such an association is represented by a line between two classes. The line terminators indicate the multiplicity of the association.

For the CompoundFormFeature class, the association with the FormFeature class represents: a CompoundFormFeature has always an association with two or more FormFeatures, whereas a FormFeature has an optional association with a CompoundFormFeature - it is only present when a FormFeature is in a CompoundFormFeature.

\subsubsection{Constraints for mutual relations}

To define the position and orientation of the instantiated form features, relations or constraints are defined 


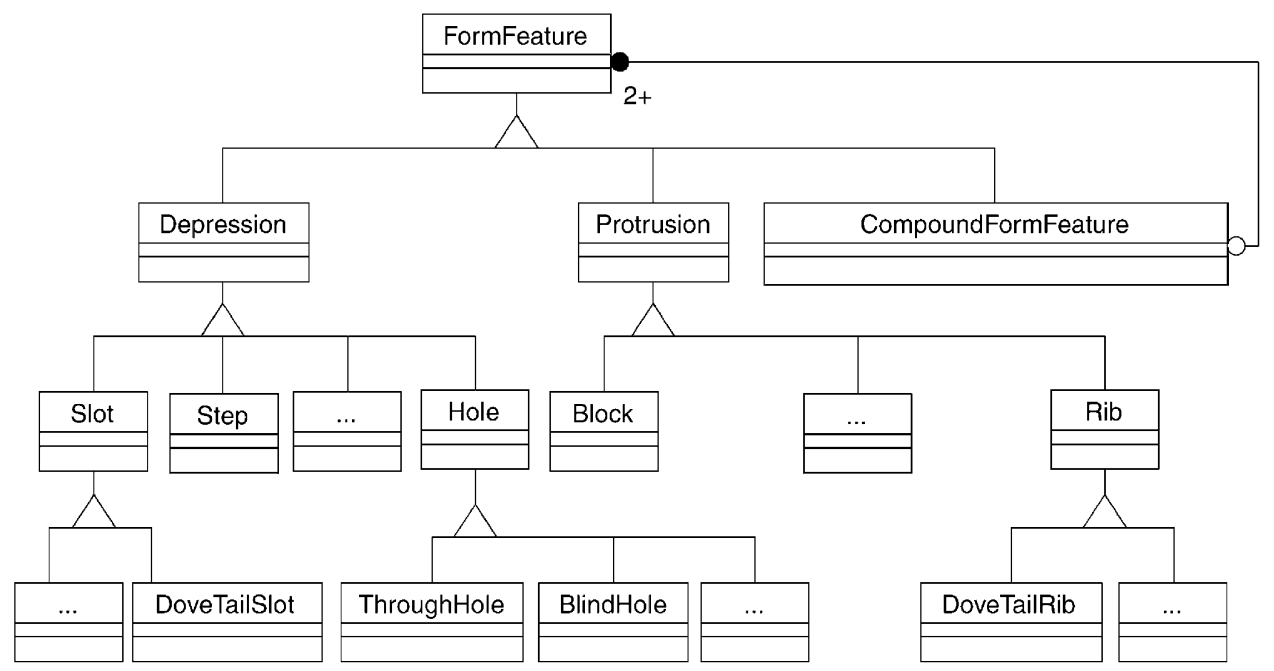

Fig. 2. Class hierarchy for form features.

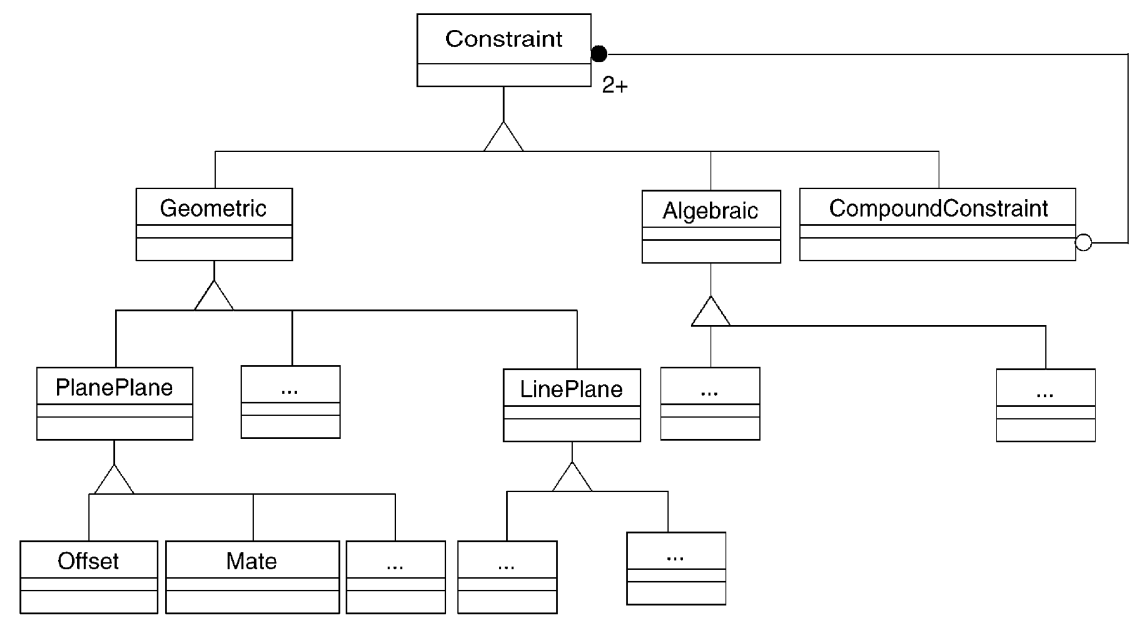

Fig. 3. Class hierarchy for constraints.

between these instances. For this a base Constraint class is defined, and derived classes to describe constraints, e.g. the Mate class for the mating relation between two planes, and the Offset class for the offset relation between two planes. Besides geometric constraints, there can also be algebraic constraints, to specify, for example, a relation between the area of a plane and the volume of a shape. An example of a constraint class hierarchy is given in Fig. 3. Again, new constraints can be defined using the CompoundConstraint class by taking combinations of other constraints. More information on constraints and how to solve them, can be found in, for example, Dohmen [6].

\subsubsection{Feature model: combining form features and constraints}

To define a complete single part, both instances of features and instances of constraints must be specified. This is done with a FeatureModel class as shown in

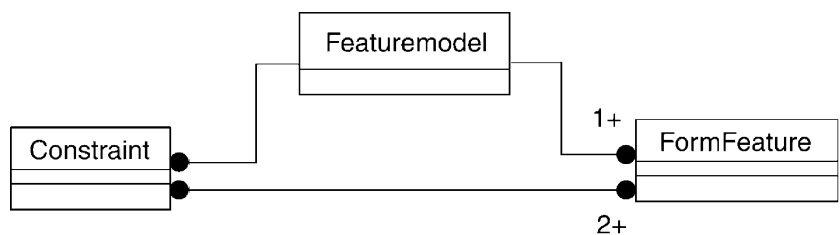

Fig. 4. A feature model representing a single part by combining form features and constraints.

Fig. 4. The FeatureModel class contains a list of feature instances, the FormFeatures, and a list of constraint instances, the Constraints on these feature instances. In Fig. 5, a feature model of a single part is shown, with instances of features and instances of constraints.

\subsection{Assembly model}

An assembly consists of combinations of single parts, where different instances can be of the same type. These 


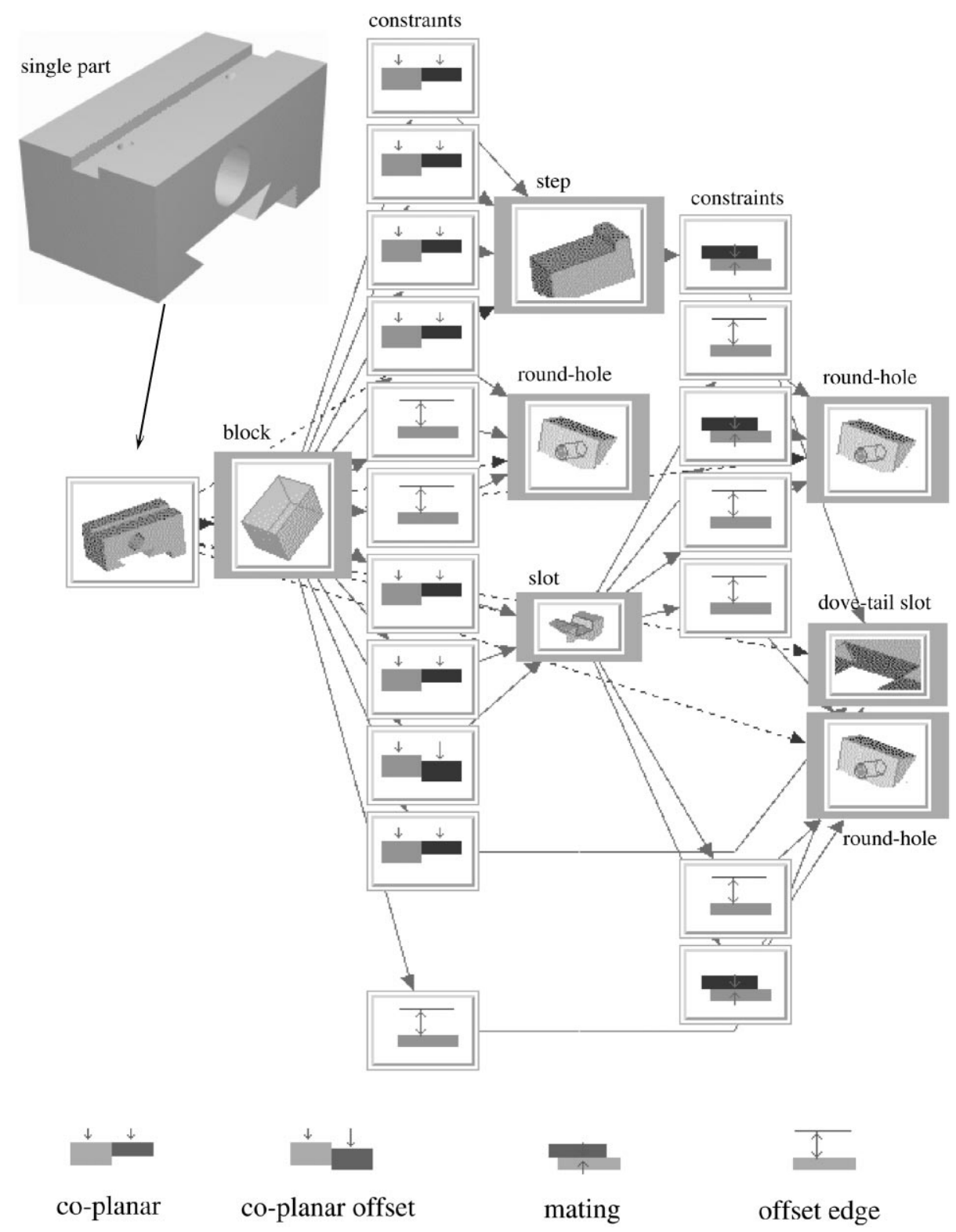

Fig. 5. Form feature model of a single part. Associations between the feature and constraint instances are shown with solid lines, associations between the feature model and its feature instances are shown with dashed lines. Note that the associations between the feature model and its constraints are not shown for clarity, although they do exist in the model.

parts are not always directly assembled into the complete product, but sometimes, and for several reasons, subassemblies are created. Both single parts and subassemblies are stable entities (with respect to motions or transportations), and can therefore be assembled onto other entities; these stable entities are called components. The already assembled components are called a partial assembly. A partial assembly can thus be a single part (when assembly has just been started), or a, possibly motion-instable, group of components (during assembly).

\subsubsection{Components as building blocks}

For each different component type, we introduce a generic component, describing the geometry by its form features. The generic component does not describe a position and orientation in the product; this is described by an instance of a generic component. In this way, a product can contain several instances of the same generic component. Each instance can have different relations within the product. Fig. 6 shows the class diagram used to describe the different components.

As can be seen in Fig. 6, there exist two derived classes from the GenericComponent class: the SinglePart and the GenericCombined class. The difference between the two is that the SinglePart inherits data elements and operations from both GenericComponent and FeatureModel, described in Section 2.1. The SinglePart class represents the feature model of a single part, which 


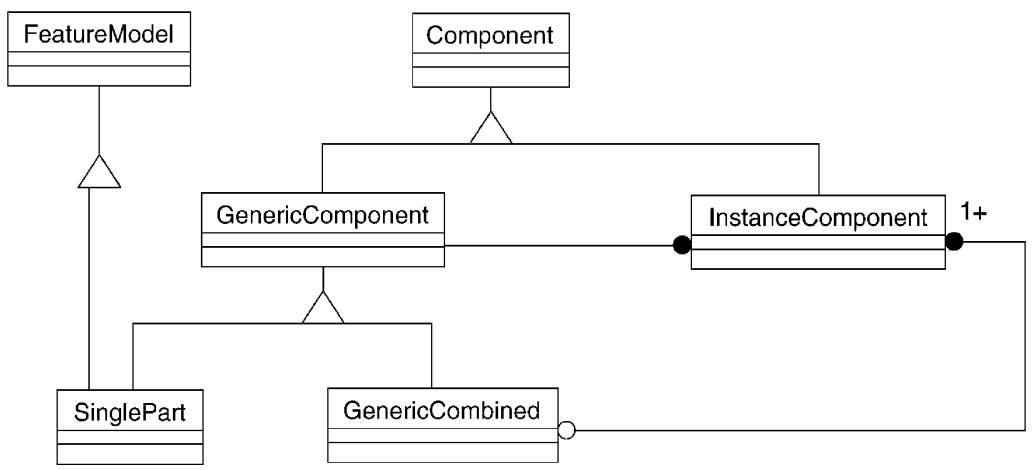

Fig. 6. Class hierarchy for different components.

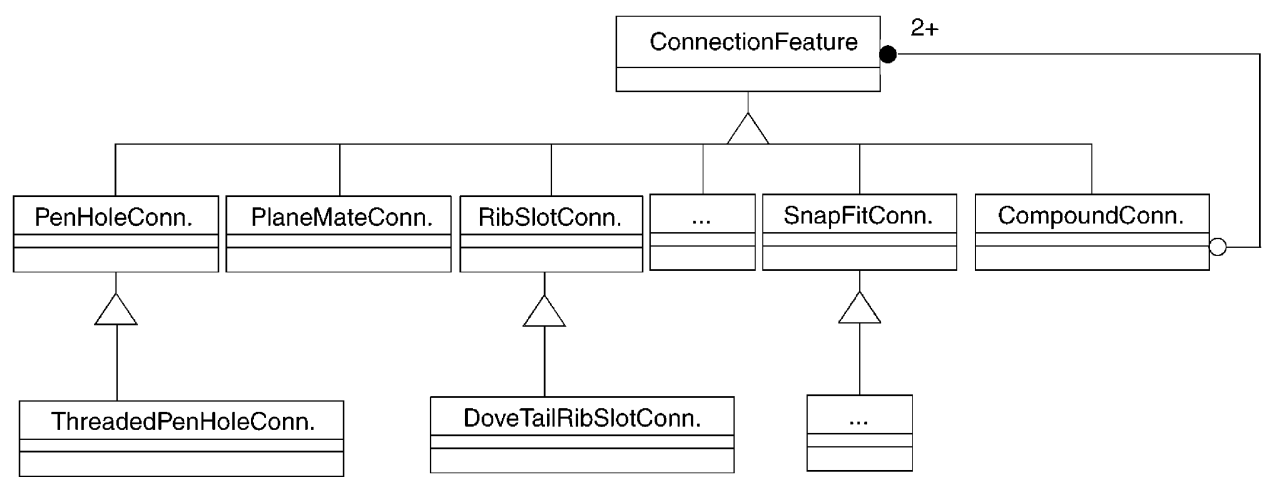

Fig. 7. Class hierarchy for connection features.

cannot be subdivided into smaller components. The GenericCombined class represents the generic components containing more than one component.

\subsubsection{Connection features for mutual relations}

As within single parts, mutual relations between instances of form features are used to specify the position and orientation of components. In the assembly model this is achieved by assembly relations, in fact the connection features that will be described in Section 3. For now it is enough to know that connection features are on a rather high abstraction level, and that there is a complete hierarchy of connection features; an example class diagram is shown in Fig. 7. The predefined set of generic connection features can be extended by using the CompoundConnectionFeature class. With this class, existing connection features can be combined to generate new connection features.

\subsubsection{Generic combined model: combining components and connection features}

The GenericCombined class represents components that consist of combinations of instances of components and connection features between them. An attribute in the GenericCombined class contains information whether it represents a stable entity, i.e. a subassembly or product, or it is not known whether the entity is stable,

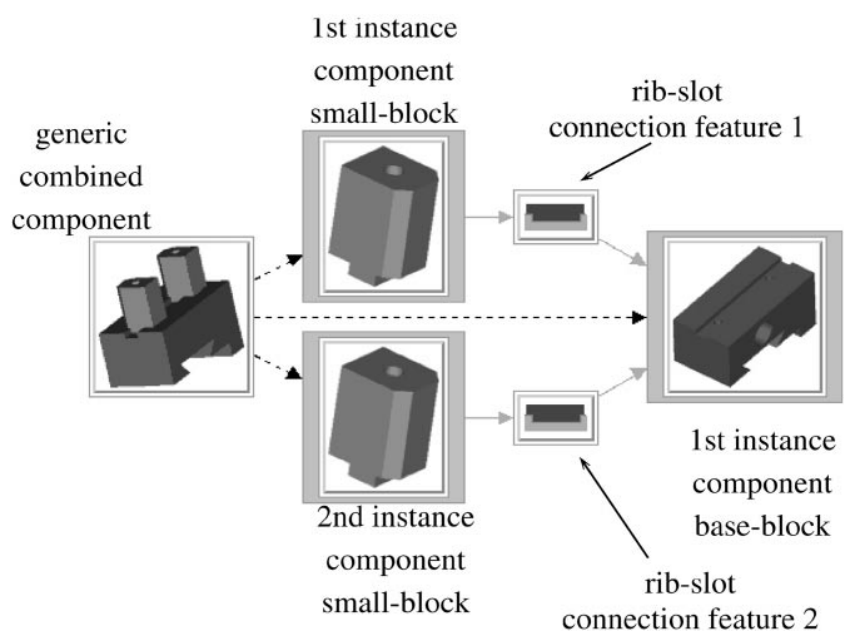

Fig. 8. Generic combined model of a subassembly.

a partial assembly. In Fig. 8. an example is given of a generic combined component, consisting of three instances of two different generic components, and two instances of a rib-slot connection feature.

\subsection{Combined product model}

As has been shown in the previous sections, in singlepart and in assembly modeling, comparable data 


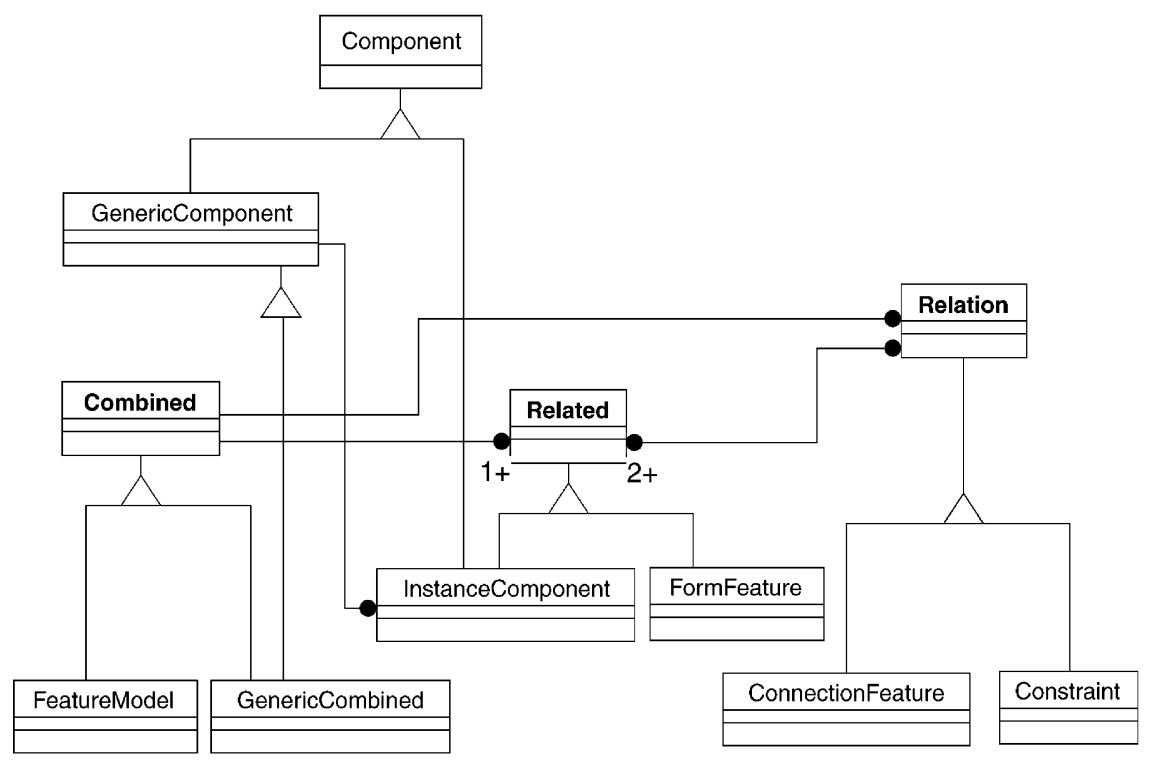

Fig. 9. Combined data structure for modeling single parts and assemblies.

structures are used to represent building blocks, mutual relations between the building blocks, and the structures of the instantiated building blocks and relations. In single-part modeling, these are represented by, respectively, instances of form features, instances of constraints, and the feature model. In assembly modeling, these are represented by, respectively, instances of components, instances of connection features, and the generic combined component.

Because of these similarities, new classes are defined to establish a uniform modeling environment for modeling single parts and assemblies. These new classes are: the Related, Relation and Combined classes, all shown in Fig. 9 with a bold face. The Related class is introduced as a base class for all building blocks, i.e. the instances of form features and the instances of components. The Relation class is introduced as a base class for all objects that represent a relation between building blocks, i.e. the constraints and connection features. The Combined class is introduced as a base class for classes in which sets of related objects and relations between them are specified, i.e. feature models and generic combined components.

Using these three base classes, a uniform way of modeling single parts and assemblies is created. This uniform modeling concept can be used to combine the two modeling environments into one environment, in which both single parts and assemblies can be modeled.

\section{Assembly features}

As within other disciplines specific information is stored in specific features, e.g. design information in design features and manufacturing information in manufac- turing features, assembly information will be stored in assembly features.

\subsection{Assembly feature definitions}

Some definitions of assembly features used in literature are the following:

- elementary relations between components [7];

- elementary relations between components extended with some assembly information [8];

- a collection of elementary relations and matching form features [9];

- an association between two form features present on different parts [10].

All these definitions are, in one way or another, focusing on the relation between components. Although the relation between components is very important within assembly, more is needed. Assembly features are therefore redefined here. An assembly feature is here defined as an information carrier for assembly-specific information. This is a broad definition, but this is needed to carry all assembly-specific information within modeling and planning.

- Assembly information can be divided into two types:

- The first type represents assembly information used to handle a component, i.e. handling-specific assembly information on generic level.

- The second type represents information about the connections between components, i.e. connectionspecific assembly information on instance level. So the assembly features are divided into handling features, representing handling information, and 
connection features, representing connections between components.

\subsection{Handling features}

A handling feature is different from other features. There are no generic descriptions of types of handling features, i.e. there does not exist some predefined set of handling feature types with corresponding behavior linked to these types. Every handling feature instance is unique and carries information on (see also Fig. 10):

- feeding, i.e. how the component is fed into the assembly system, e.g. by component trays or feeders;

- fixturing, i.e. how the component is fixed (only for base components);

- grippers, i.e. which grippers can be used to grasp the component, and how this can be done;

- matching grasping areas, i.e. for each gripper all areas where it can grasp the component, independent of the actual position and orientation of the component.

The HandlingFeature class provides methods to store and retrieve information about feeding, fixturing and grasping, for a generic component. The feeding and fixturing information consists of predefined position and orientation information, together with involved contact areas. The gripper information consists of, among other things, the number of fingers, the maximum finger width, the finger length, minimal and maximal grasp forces, and available motions.

\subsection{Connection features}

A designer thinks in higher-level connection types between components, rather than in elementary rela- tions. These connection types can be structured in socalled connection features, which are much closer to his way of thinking than the elementary relations.

The idea of connection features is that characteristics of connection types can be incorporated in these features. By specifying a connection feature in a product model, the assembly-specific information known by the feature is also available in the model. Characteristics of a connection are, of course, the static characteristics when the connection has been established, but dynamic characteristics are even more important, especially within assembly planning. These dynamic characteristics are about how the connection can be established (during assembly) or can be broken (during disassembly).

The ConnectionFeature class provides methods to store and retrieve assembly information for a specific connection between several components. Here an extendible list is provided, to show some of the information that is available when the connection type is known:

- Involved form feature types. The type of the form features involved in the connection, e.g. both the rib and slot feature in a rib-slot connection;

- Final position. The final position, or goal position, is the position and orientation of the assembled component relative to the partial assembly, after the assembly operation has been completed. This position can also be specified by the relative position and orientation of the involved form features;

- Insertion position. The insertion position is the position and orientation relative to the final position where the insertion operation starts. At this position, there is just no contact between the component to assemble and the partial assembly to assemble it on;

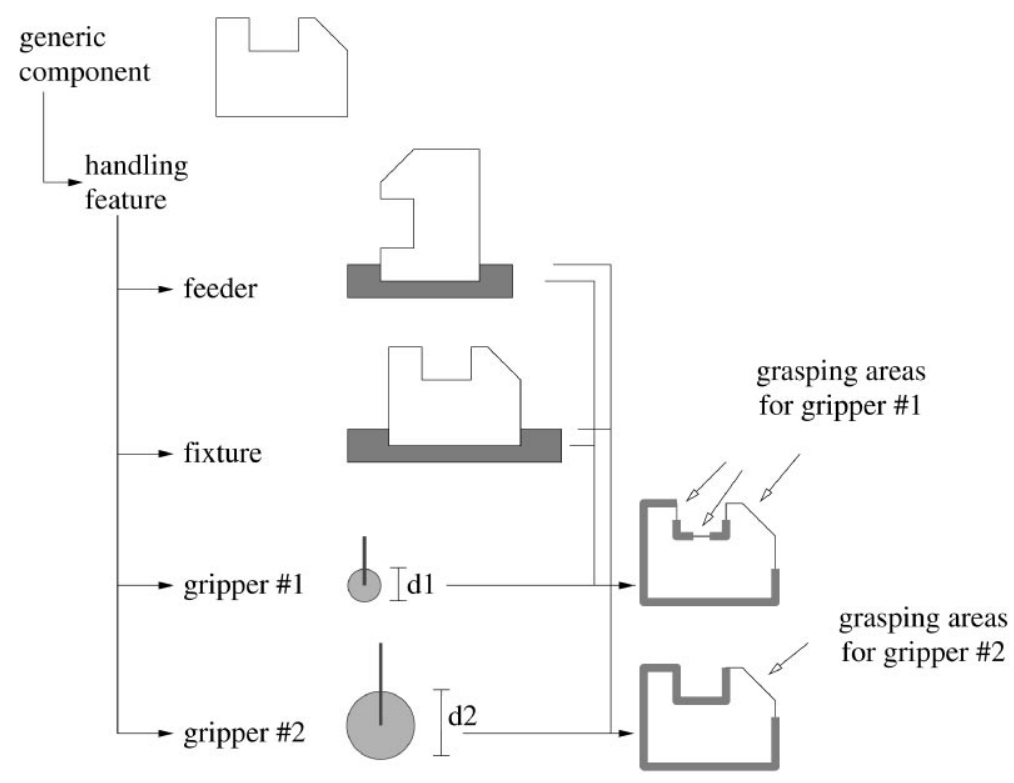

Fig. 10. Attributes of handling feature. 
- Insertion path. The insertion path is a trajectory from the insertion position to the final position. This trajectory can also be a predefined procedure for some compliant motion, i.e. a motion using contacts during assembly to eliminate uncertainties in position and orientation;

- Tolerances. Tolerances required to establish the connection between the components;

- Contact areas. Contact areas are the areas involved in the connection;

- Internal freedom of motion. The internal freedom of motion is the set of motions that can separate the component and the partial assembly. This can be both translational and rotational freedom of motion;

- Geometric refinements. Geometric refinements are special refinements to ease the assembly operation, e.g. rounds and chamfers.

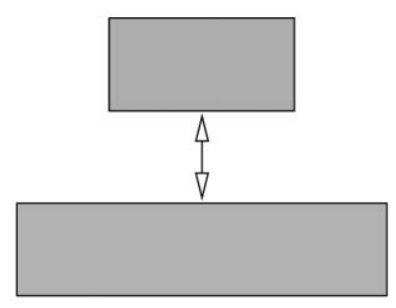

(a)

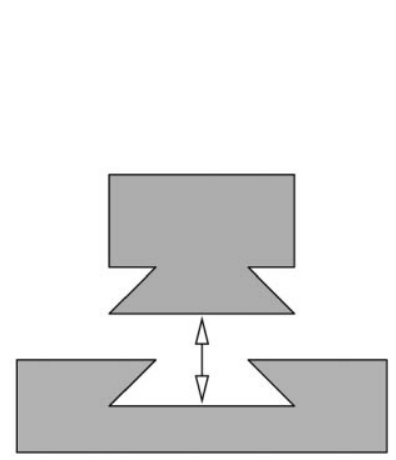

(c)

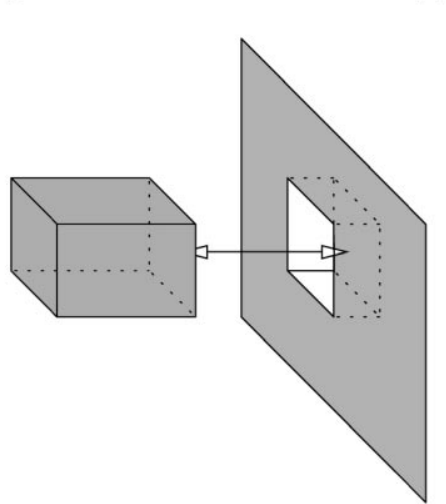

(e)

Fig. 11. Examples of elementary connection features. (a) Plane mate connection; (b) rib-slot connection; (c) dove-tail connection; (d) penhole connection; (e) rectangular pen-hole connection.
A predefined class hierarchy of commonly used connection features, the elementary connection features, is shown in Fig. 11. It is possible to extend the used set by defining completely new ones, but it is also possible to create new connections by combining already existing ones. The latter can be done by using the CompoundConnectionFeature class. Examples of compound connection features are given in Fig. 12.

A special type of connection is the attachment. Attachments are connections enforced by so-called agents. Agents, consisting of components and connections, are only needed to enforce a connection, see also Sanderson et al. [11]. Take, for example, a functional connection to fix two plates. This can be done by taking a bolt attachment connection. So, by specifying the functional connection to fix two plates, a new component is introduced with its own connections, i.e. the bolt with its mutual connections. The new components and mutual connections are called the agents - divided into component and connection agents. By choosing a different connection to

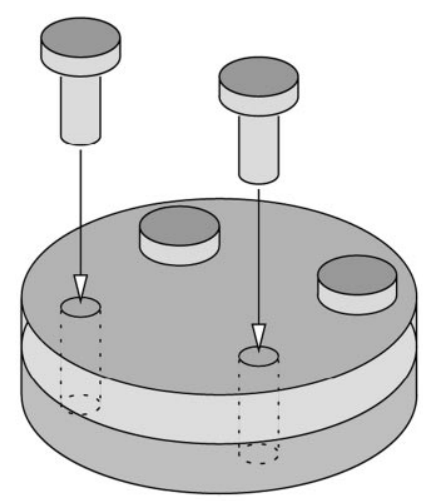

(a)
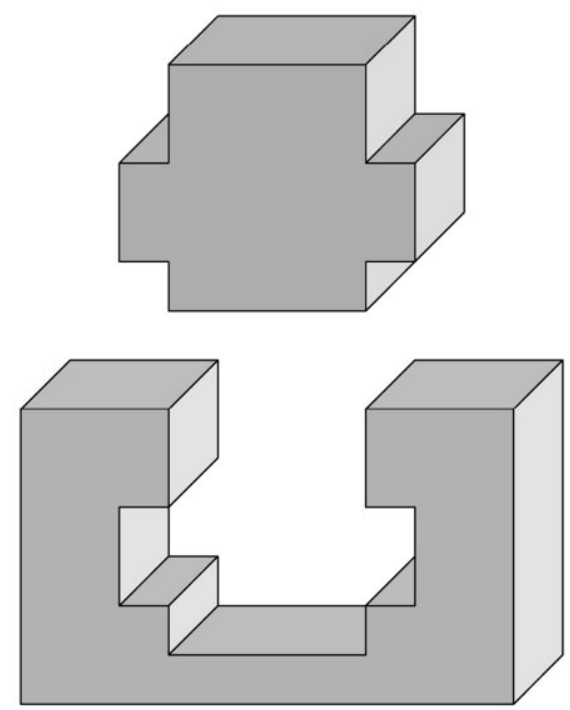

(b)

Fig. 12. Compound connection features. (a) Compound circular pattern connection feature; (b) compound rib-slot connection feature. 
fix the plates, e.g. bolts and nuts to fasten them, other component and connection agents are introduced.

The fact that a component or a connection is an agent, is important during modeling. It provides information why a certain component or connection is in the model: the mutual attachment connection is needed. Deleting an agent from a model will therefore have direct influence on the attachment relation. Because an attachment is always related to at least one component agent, by instantiating an attachment, always one or more specific components are instantiated.

\section{Assembly modeling}

A prototype assembly modeling system has been implemented. In this section, this system will be shortly described. First, some implementation issues are given, and then the most interesting aspect of the system, combined class modeling, is discussed.

\subsection{Implementation issues}

The architecture of the prototype is shown in Fig. 13. Modeling and planning algorithms make use of the same product model, the feature-based product model described in Section 2. There is one interface module, making it possible to call planning algorithms during modeling and vice versa, which is useful for DFA analyses. Some of the functions can be accessed by a graphical user interface (gui), to provide the user with an easy mechanism to manipulate the model. Other applications, which do not directly make use of the graphical user interface, can also have access to the interface for modeling and planning functionality.

The system is able to create complete components and assemblies from the provided instance related and relation objects. A simple constraint solver has been implemented to generate the position and orientation of every instance related object. The complete geometry of the components and assemblies is represented with the ACIS geometric library. Once a part or product has been created, it can be directly used as a generic component by instantiating it into another partial assembly.

In Section 2.3, a description was given of the Combined class structure, which provides a uniform modeling environment for single-part and assembly modeling. From Combined objects, there are association relations to both Related and Relation objects. There are also association relations between Related and Relation objects themselves. All theses objects and their mutual associations can be represented by a graph; the nodes are the objects, the arcs the associations. On the other hand, the Combined object itself represents some geometry, i.e. a single part or an assembly. Therefore, the same object can be looked at in two different ways: as a graph or as geometry. For this, a geometry viewer and a graph viewer are used. A geometry view consists of a line drawing or a shaded image of the model.

An example of a graph view is shown in Fig. 14. For building and viewing the graph, the XHDG widget set [12] has been used. This widget set is capable of representing multi-level graphs. One can hide details of a graph at a lower level, by representing the complete graph as a single node at a higher level. By clicking on such a multi-level node, it will be expanded, and the underlying graph is shown.

Both types of views have advantages and disadvantages, and used together they can supplement each other. A geometry view can provide a good spatial notion of the model, whereas a graph view can provide a good insight in the structure of the model and a good selection mechanism for both Related and Relation objects.

The planning modules can be directly accessed during modeling, both in a graph and in a geometry view. This makes it possible to perform DFA actions during modeling.

\subsection{Combined class modeling}

In this section, possible ways of creating and maintaining combined models are described. Basically there are two ways for this: off line by describing the model in a text file and parsing the file into the modeling system, and on line by using the graphical user interface of the system. There is no need to further describe the steps taken in off-line modeling using a text file - it works as with any other interpreter. On the other hand, some concepts of on-line modeling warrant further explanation.

Before the actual creation of the combined model can start, the generic elements needed in the structure must be available. These elements can be separated into the

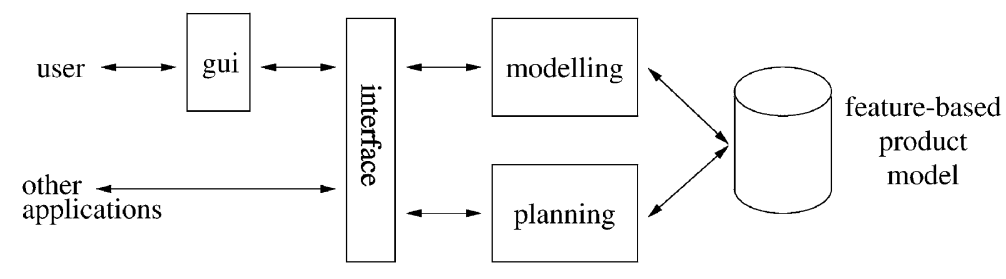

Fig. 13. Prototype architecture. 


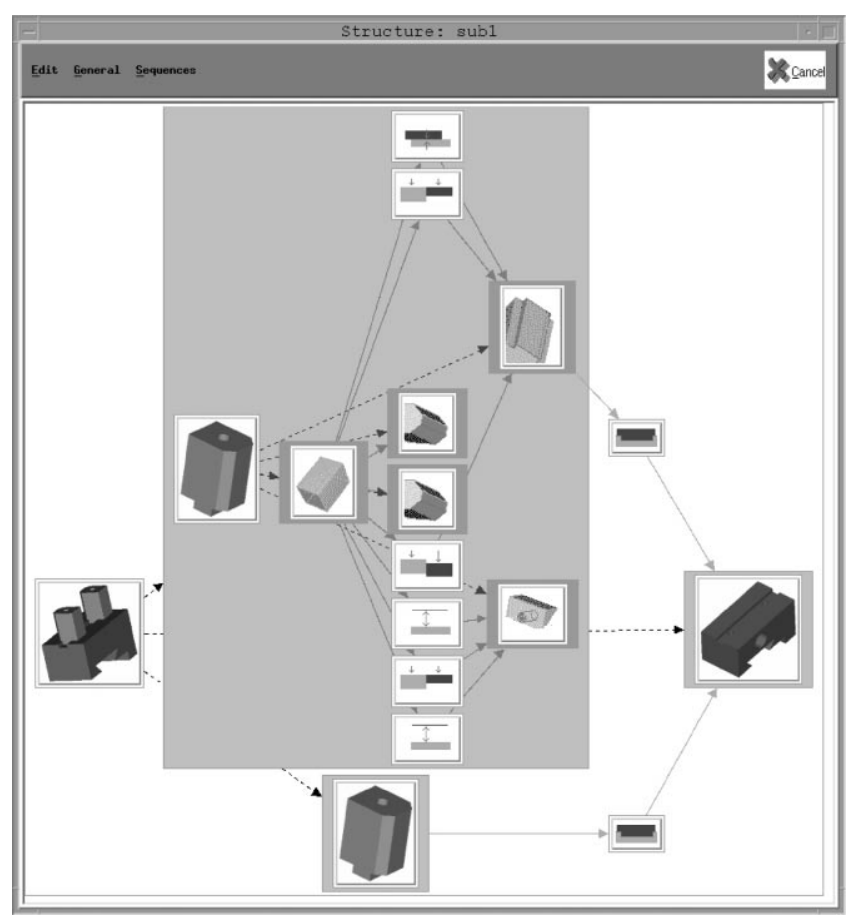

Fig. 14. A node can be expanded to show its underlying model; note that associations are now shown on form feature level.

related and relation elements, and depending on the fact whether one wants to create a feature model or an assembly, the form features and constraints, or the generic components and connection features are shown.

For example, before one can create a feature model, form feature descriptions and constraints must be available in some kind of library. These can be selected and added to the feature model. Then associations between the form features and the constraints must be defined in such a way that the result will be the required feature model.

An element added to the model can be an object from the Related or the Relation class. Depending on which type one first adds to the model, there are two ways of modeling: related-driven modeling and relation-driven modeling.

In related-driven modeling, first instances of Related objects, generic form features or generic components, are added, and then Relation objects between these Related objects are specified. The only extension of this concept to the commonly used bottom-up concept, is that both Related and Relation objects contain more knowledge about each other, because of the features. For example, according to already specified Related objects, a selection can be made of possibly matching Relation objects. This can be helpful for the designer in specifying the right relations between related objects. A disadvantage is that this is still a bottom-up modeling concept: the detailed generic objects must already be available before the relations are specified.
In relation-driven modeling, on the other hand, first Relation objects are specified, and then Related objects. At first sight, this does not differ much from the previous way, e.g. the Related objects can be selected from a matching list dependent on the already chosen Relation objects. This is still bottom-up modeling. However, the relation-driven modeling concept can be helpful in realizing a top-down modeling concept. Suppose that the user is capable of specifying some undetailed Related objects. Then, by specifying Relation objects between these instances, matching geometry can be created on the Related objects, because of the knowledge available in the Relation objects. In this way, more detail is specified for the Related objects. This is modeling from the conceptual towards the detailed level, i.e. top-down modeling.

It is possible to use the two modeling techniques alternately in one modeling session. Some parts of the model can be modelled by using the related-driven technique, e.g. when using standard components. Other parts of the model, which are more dependent on preferred connections, can be modeled by using the relation-driven technique.

\section{Assembly planning}

Assembly features cannot only be effectively used in modeling, but also in planning. Within assembly planning, several modules exist that can only work properly with certain assembly-specific information. How assembly features can provide such information, is described in this section.

In literature, the term assembly planning is often exclusively used for assembly sequence planning [13-15]. Although assembly sequence planning - finding feasible sequences in which the product can be assembled - is a very important planning module in assembly planning, there exists many other modules [16]. Assembly sequence planning is highly dependent on these other modules, especially where these modules concern assemblability.

The following list briefly enumerates the most important assembly planning modules with their specific goals [17]:

- Fixture planning. A fixture planner determines the base component, the first component to assemble, and the fixtures;

- Feeding planning. A feeding planner determines the feeders for the components to assemble;

- Stability analysis. A stability analyzer checks whether an assembly is stable, and thus can be used as partial assembly or subassembly;

- Grip planning. A grip planner determines the tools for gripping a component, and the areas on the component where to grasp it; 
- Subassembly planning. A subassembly planner tries to divide the complete product into subassemblies. The main requirement for subassemblies is that they remain stable when manipulated, but other requirements can be that the subassembly must fulfil some function, or is important for service purposes;

- Motion planning. A motion planner determines a path for a component to be added to the partial assembly, from the feeding position to the final assembled position;

- Assembly sequence planning. An assembly sequence planner determines feasible assembly sequences for a product;

- Scheduling. A scheduler determines an optimal assembly sequence for a complete batch of products.

In the remainder of this section, it will be indicated how assembly features can be profitably used in stability analysis, grip planning, motion planning and assembly sequence planning.

\subsection{Stability analysis}

Stability analysis is a very important issue in assembly. For example, in subassembly planning, an assembly is decomposed into stable subassemblies, and in assembly sequence planning, stable components must be assembled onto stable partial assemblies. According to Boneschanscher [18], three types of stability can be distinguished, depending on the forces taken into account:

- Gravitational stability, only takes into account gravity,

- Assembly stability, takes into account both gravity and additional forces caused by the joining operation,

- Motion stability, takes into account gravity and additional forces due to acceleration during motion of the assembly.

The focus will here be on the first stability type, gravitational stability. Therefore, where the word stability is used, gravitational stability should be read. The other types of stability can be checked using the same methods as for gravitational stability, by using the resulting force instead of the gravitational force. However, the exact calculation of the resulting force can be very difficult. To analyze whether an assembly is stable, two steps can be distinguished: first analyze translational stability, and then rotational stability. Within translational stability analysis, translations between components are taken into account (see Fig. 15(a)), within rotational stability analysis, rotations between components (see Fig. 15(b)). The latter step is far more complex than the first. One advantage of dividing the stability analysis into these two steps is that the second step can be omitted when the first step already finds the assembly to be instable.

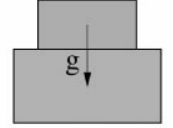

stable

(a)

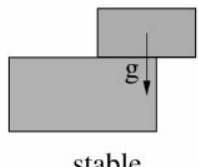

stable

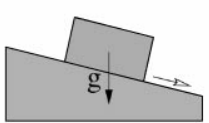

instable

instable

(b)

Fig. 15. Translational (a) and rotational stability (b)

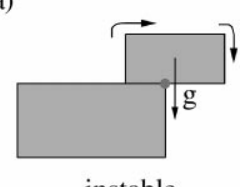

\subsubsection{Translational stability}

During assembly, each time a component is added to the partial assembly, and a relatively easy thing to analyze is whether a just added component has some stability impact on the new partial assembly. Taking friction into account, the just added component on a partial assembly can have stability effects on other components already assembled. However, here friction is not taken into account, and therefore only the component just added has to be checked for stability.

To know whether a component can translate under influence of the gravity, one has to investigate whether it is possible to translate, or move, the component in the gravitational direction. For this purpose, the internal freedom of motion (IFM) of a component is used, the set of motion directions that can separate a component from its partial assembly [19]. If one of the directions in the IFM can be transformed in such a way that it contains an element in the direction of the gravitational force, then the component is instable in relation with its partial assembly.

Lee and $\mathrm{Yi}$ [19] used in their definition of IFM only the three principal axes, which is too restricted: in that case assembly is only possible in these three directions. To allow all directions, a method called visibility mapping is used. This method is also used by Chen et al. $[20,21]$ for several applications in manufacturing, such as computing machinability on $\mathrm{CNC}$ machines, and mould and die design. Mattikalli and Khosla [22] also used this method for stability analysis.

Traditional visibility mapping methods map the possible translation directions onto a unit sphere, resulting in a so-called visibility map or VMap. Computing with such Vmaps, in particular taking the intersection of two Vmaps to determine the set of motions that can separate a component from two other components, turns out to be difficult. Therefore, Mattikalli et al. [23] and van Holland and Bronsvoort [24] independently developed an alternative cube representation for the IFM that is computationally more attractive. 


\subsubsection{Using connection features for internal freedom of motion}

An advantage of using connection features, is that there is already knowledge of possible motion directions available within the generic connection. Every generic connection feature contains the generic contact areas, and a generic description of the IFM. For every instance, this generic IFM can be directly used to retrieve the actual IFM. The IFM of a component can then be computed by intersecting the IFMs of all involved connection features. To determine translation stability, the resulting IFM must be intersected with an IFM representing a plane with the normal in the direction of the gravitational force. If this intersection is empty, the assembly is translationally stable, otherwise it is translationally instable.

\subsubsection{Rotational stability}

To decide that a component is rotationally instable, one has to find a rotation axis around which the component can rotate, given the available forces. A rotation axis differs from a translation direction in that an axis contains both a position and a direction, which makes the computations even more difficult. Another difference is that when already more than two components are in the resulting partial assembly, one has to take into account that already examined contact areas can become instable by adding a new component. This can be seen in Fig. 16, where assembling a component $\mathrm{C}$ results in a rotationally instable connection between component $\mathrm{A}$ and $\mathrm{B}$, al-

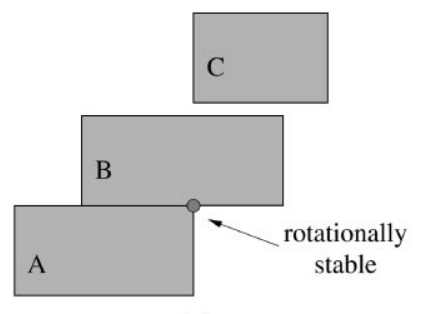

(a)

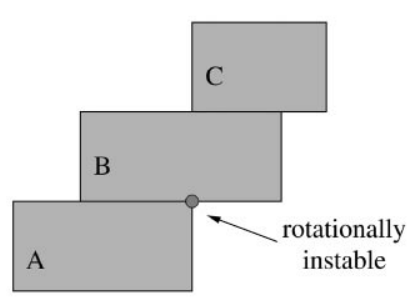

(b)

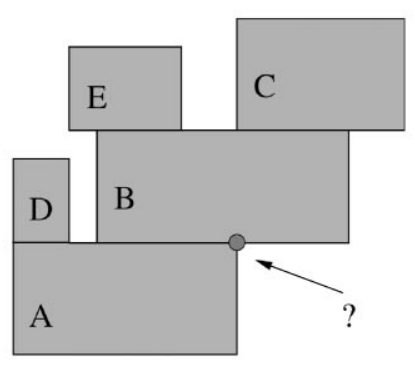

(c)

Fig. 16. Assembling a component can change a previously stable connection into an instable one. (a) Rotationally stable connection between components A and B; (b) rotationally instable connection between components $\mathrm{A}$ and $\mathrm{B}$; (c) rotational stability of the connection between $\mathrm{A}$ and $\mathrm{B}$ is dependent on $\mathrm{C}$ and $\mathrm{E}$, but not on $\mathrm{D}$. though the connection between component $\mathrm{B}$ and $\mathrm{C}$ itself is rotationally stable. Also other components not directly connected to the just assembled component, can have influence on the stability of the assembly, e.g. component $\mathrm{E}$ in Fig. 16(c). These components must also be considered.

Mattikalli and Khosla [25] described a theoretical basis for finding all possible rotation axes, the rotational degrees of freedom. In particular, they showed that it is sufficient to investigate the edges of the convex hulls of the contact areas involved. They also developed a structure to represent all possible rotation axes.

\subsubsection{Using connection features for rotational degrees of freedom}

By using connection features, there is no need to investigate all edges just mentioned. Take, for example, the rib-slot connection feature. This feature already contains the information that there are at most four possible rotation axes. The possible rotation axes are stored within the generic descriptions of the connection features. Using information available on instance level, the actual possible rotation axes can be directly provided, see Fig. 17. By combining all possible rotation axes of all involved connections, the resulting possible rotation axes are found. These can be checked against external force information, to determine whether the assembly is rotationally instable.

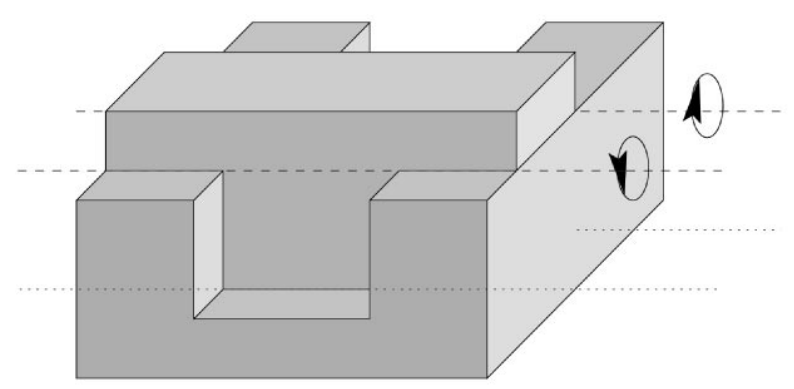

(a)

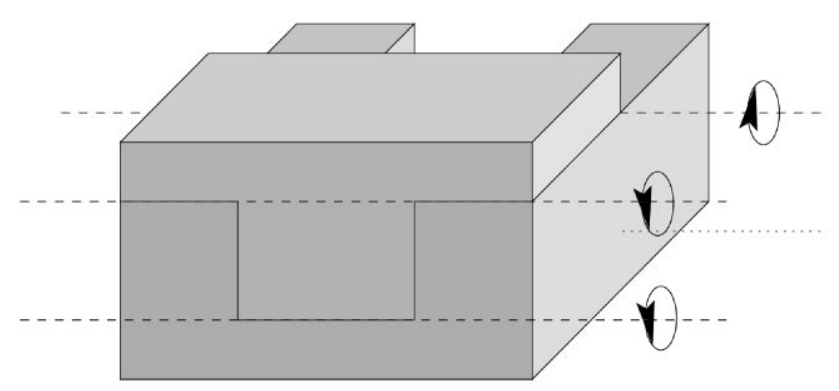

(b)

Fig. 17. Connection features can provide possible rotation axes, by combining available generic and instance information. (a) This instance has two of the four possible rotation axes; (b) this instance has three of the four possible rotation axes. 


\subsection{Grip planning}

The main purpose in grip planning is to find how a component can be grasped, in such a way that it can be assembled onto the partial assembly. To find a set of grips, all areas where the fingers of a certain gripper can be positioned must be found, the so-called finger domains. This is a time-consuming step. When all finger domains have been determined, a selection must be made so that a stable grip is found. When the gripper has only two or three fingers, the positions to place these fingers are always on a line, respectively in a plane, called the grip plane. This reduces the problem of finding stable grips from a $3 \mathrm{D}$ to a $2 \mathrm{D}$ problem. The usual way to find the finger domains is by retrieving the required information from the geometry of the product model only.

\subsubsection{Using geometry only to find finger domains}

Finger domains can be found by taking the complement of all areas where the fingers cannot be positioned, i.e. the non-free regions. One method to find these regions is described by van Bruggen et al. [26], using so-called Expanded Face Solids (EFS). Baartman [27] has described some extensions to this method. The EFS for a face in the product model is an envelope volume dependent on the thickness of the finger tip.

To get all non-free regions in the model, an EFS for every face in the model must be created, and intersected with the model. Because this has to be done for every face, the time complexity of this method is dependent on the squared number of faces.

\subsubsection{Using features to find finger domains}

The idea of using features in grip planning is to interrogate the features in a product model to see where they can or cannot be grasped. Using feature information will speed up the finger domain determination. Which types of features are used, can be seen in Fig. 18. In the first phase of the finger domain determination, form features and handling features can give information about finger domains. This set, initially the same for every instance of

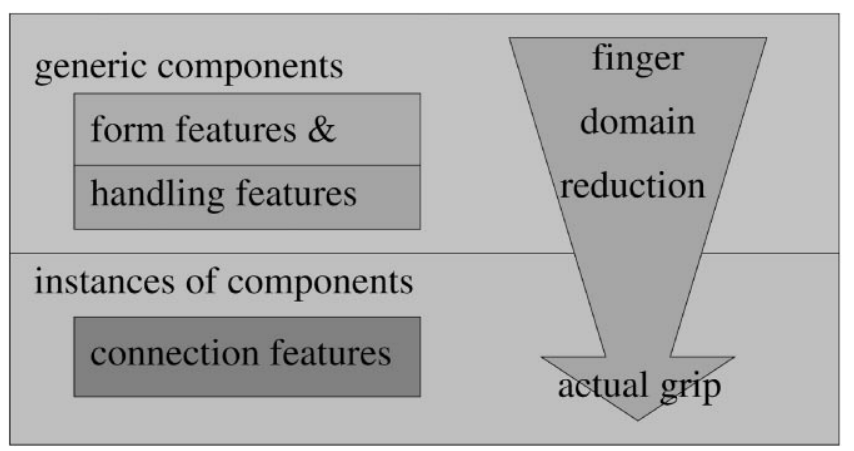

Fig. 18. Features used to speed up the finger domain determination, and to provide additional information for the actual grip. the component, can be used in the second phase to determine actual gripper-specific grips on instance level. The latter can be done by combining the initial set of finger domains with connection information and assembly directions stored in the connection features.

\subsubsection{Using form features}

In every generic form feature definition, information useful for grip planning can be stored. By using this information, every instance can generate potential finger domains from its parameters. In Fig. 19, information stored in the generic definition of a dove-tail slot feature is shown. The parameters for width, length and depth are defined here. On the basis of the values for the parameters of an instance, the non-free regions can be computed for a specific finger tip. The influence of other features on this feature, which can further restrict the free regions, is not yet taken into account, and therefore the found finger domains are called local finger domains.

The local finger domains on each feature can be enhanced by giving some kind of ranking to it, depending on the graspability known by the feature. Curved faces can be used for grasping, but it is better to use planar faces. Some finger domains can be reached by the gripper from both top, and front and back side, other finger

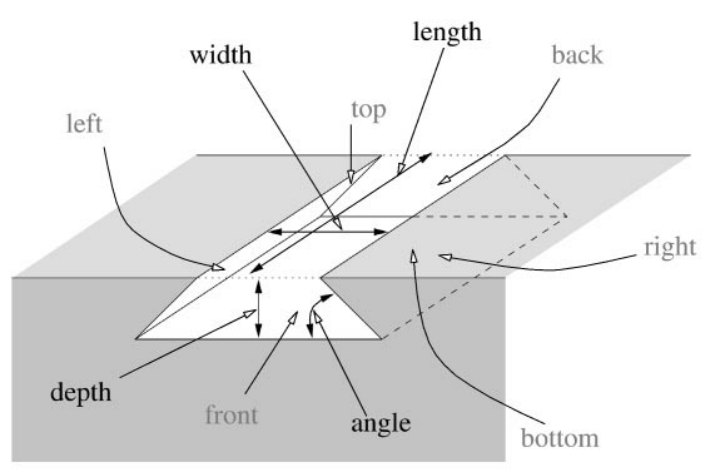

(a)

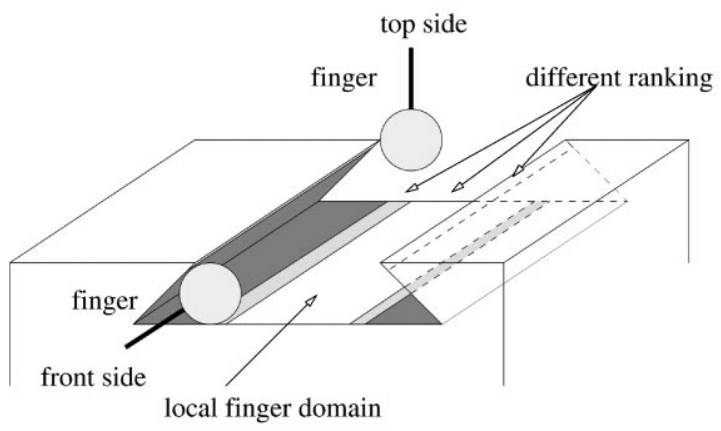

(b)

Fig. 19. Generic definition of a dove-tail slot feature, and an instance of it with its local finger domain (different shadings represent different rankings: light shading for areas that can easily be grasped, dark shading for areas that cannot be grasped). (a) Generic definition of form feature dove-tail slot; (b) instance of dove-tail slot with local finger domains dependent on gripper parameters. 


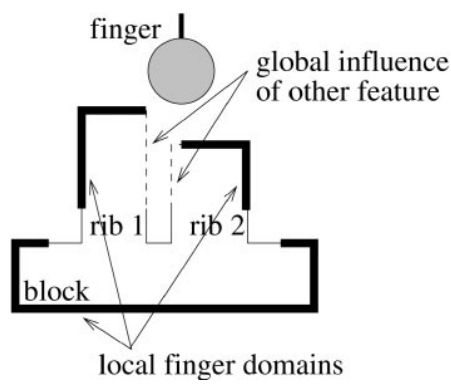

Fig. 20. Features can have influence on other features, reducing their finger domains.

domains can only be reached from front and back side, resulting in a lower ranking because the orientation of the gripper must be taken into account here. See Fig. 19 for an example of different rankings. The ranking can be used later when the best grip must be chosen from all possible grips. The local finger domains found for all features in the model can be combined to determine the local finger domains for the complete model.

Areas found to be within a local finger domain, can still be non-free regions because of the influence of other features. A feature does, in general, not know, whether there exists influence from other features. For example, in Fig. 20, the two rib features may be too close to each other to position a finger in the slot between them. Therefore, an additional step has to be executed to compute the global finger domains from the already generated local finger domains. For this, a variant of the geometric EFS method can be used. Fortunately, features themselves can give some information on whether they can possibly influence the finger domains of each other. Only additive features, features that add volume to a model, can have influence on finger domains of other features, subtractive features cannot.

\subsubsection{Using handling features}

Relevant information stored in the handling features can be used by the form features, e.g. the finger width of a specific gripper is used by the form features to generate the global finger domains for that gripper. Besides gripper information, also feeder and fixturing information can be found in the handling feature. This information can be used to further reduce each gripper-specific set of global finger domains found for every generic component. All areas involved in feeding and/or fixturing the component are non-free regions, and therefore removed from the set of global finger domains. The resulting set of finger domains is stored for every type of gripper in the handling feature. This gripper-specific set of finger domains can be used for every instance to determine the actual grip.

\subsubsection{Using connection features}

Until now, only information has been considered independently of the position where the component will be assembled on the partial assembly. This position is different for every instance of the component. Additional useful information can be retrieved from the connection features in the model. These features 'know' which areas cannot be in the set of finger domains because they are involved in a connection. These areas are removed from the set of finger domains.

To find stable grips, features can be used as well. Possible grips can be generated by slicing the component perpendicular to the assembly direction. The slice reduces the problem of finding an actual grip from a 3D problem to a $2 \mathrm{D}$ problem, or a $2 \frac{1}{2} \mathrm{D}$ problem if the orientations of the faces are taken into account. The global finger domains are intersected with the slice, and the resulting intersection lines are used to determine possible grips that have correct values for closure, equilibrium and stability.

The form features can be further used to retrieve information on possible grip edges in such a slice. From an edge, the related face and form feature can be found. This feature can give information on the opposite face, which can be used to find a correct closure. For example, a block feature 'knows' that its back face is opposite to its front face, and that they are parallel. Finding opposite faces can, in general, be used as a heuristic to find possible grips. Another heuristic can be to first evaluate the finger domains available on the largest design feature in the model.

See van Holland and Bronsvoort [28] for more details on this feature-based grip planning method.

\subsection{Motion planning}

Motion planning consists of two steps (see Fig. 21). In gross motion planning, a collision-free path is searched for a component from the feeding position to a position near the partial assembly, the insertion position. In fine motion planning, the last phase of the assembly, the actual insertion of the component, is taken into account: an assembly path is searched from the insertion position to the final position. Here contacts between components, by definition, cannot be avoided.

\subsubsection{Gross motion planning}

To determine the gross motion path, the geometry of component and partial assembly are taken, and an obstacle-free path is computed in 3D space.

Within gross motion planning, there is no major benefit of using assembly features. Only for determining the insertion position, the position where gross motion ends and fine motion starts, assembly features can be useful.

\subsubsection{Fine motion planning}

In fine motion planning, the contact areas between component and partial assembly are used to determine a path that 'leads' the component to its final position. 


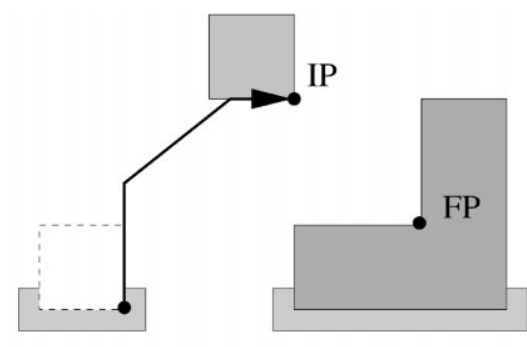

feeder

fixture

(a)

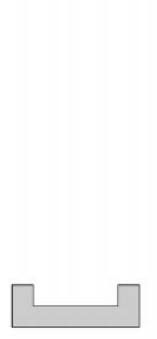

feeder

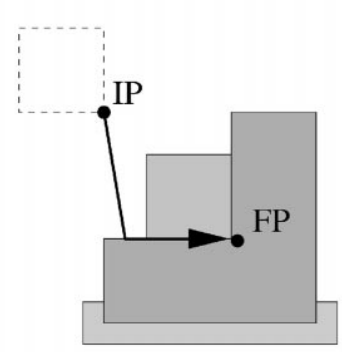

fixture (b)

Fig. 21. Motion planning consists of gross and fine motion planning. Note that for clearness the distance between IP and FP has been somewhat enlarged in this example. (a) In gross motion planning, the component is moved from the feeding position to the insertion position (IP), avoiding collisions; (b) in fine motion planning, the component is moved from IP to the final position (FP) by using contacts to reduce positioning uncertainties.

Assembly features, in particular connection features, can be profitably used in two ways: by providing possible motion directions and possible fine motion strategies. The internal freedom of motion stored within connection features, can be used to retrieve the possible motion directions.

Specific connections, e.g. (threaded) pen-hole and plane-mate, often have their own specific fine motion strategies. To select a suitable strategy in case no connections features are used, the type of connection must be retrieved from the geometry, which is sometimes very hard, or even impossible. When using the contact information stored in connection features, the type of contact is already available. In addition, generic fine motion strategies can already be stored within the generic connection features. When there is a need for a specific fine motion, the specific instance of the connection feature can provide the generic fine motion strategy with parameters, resulting in the fine motion strategy to be used.

\subsection{Assembly sequence planning}

There exist many publications on assembly sequence planning. For an overview, see Gottschlich et al. [17]. Important references are De Fazio and Whitney [29],
Homem de Mello and Sanderson [13], Lozano-Péres and Wilson [30] and Chakrabarty and Wolter [31].

The whole process of assembly sequence planning can be subdivided into three main steps, of which sometimes the first two steps are taken together:

1. generate precedence relations between the components of a product,

2. generate all feasible assembly sequences,

3. find the optimal assembly sequence from the feasible sequences.

These three steps are now briefly described.

\subsubsection{Generate precedence relations}

Sometimes the precedence relations are gathered by interrogating a human assembly planner, but this is extremely difficult for larger models, because of the large number of precedence relations. Therefore, mostly computer tools are used. These tools take as input a geometric description of the product, with the available relations between components. The - mostly low-level - relations are restricted to whether components mate with each other, and whether these matings are fixed. In the previous sections, it has already been described how features in general, and assembly features in particular, can be used to determine certain precedence relations. For example, stability analysis uses assembly features to determine the stability of a component. Only a stable component can be assembled, so this induces certain precedence relations. Using features for generating precedence relations does not change the basic ideas on how to generate them, but it differs in the way assemblyspecific information is retrieved. Feature models can significantly decrease the complexity and the time used to retrieve this information.

\subsubsection{Generate feasible assembly sequences}

Feasible assembly sequences are those sequences that can result in the complete product. The precedence relations are used to generate feasible sequences. The feasible sequences can be represented by an AND/OR graph [32]. See Fig. 22 for a simple product, and Fig. 23(a) for its AND/OR graph. Mostly the AND/OR graph is built simultaneously with investigating the precedence relations. This can be done by first searching for feasible disassembly sequences for the product, and then reversing them to get the feasible assembly sequences. Every component in the product is investigated in turn, to check whether it is possible to disassemble it from the product. If the component can be disassembled, it is removed from the product, and the procedure is repeated on the components left, until all components have been taken out. Finding disassembly sequences is combinatorially less expensive than finding assembly sequences, because every step in disassembling a product leads to a smaller product that always can be disassembled, 
whereas every step in assembling a product leads to a bigger product, with increasing chances on constraining precedence relations for components still to assemble. The latter can result in checking many infeasible sequences.

\subsubsection{Retrieve the optimal assembly sequence}

The optimal sequence - or semi-optimal sequence, because the optimal can seldom be found due to all kinds of competing criteria [29] - is the sequence with the optimum for some kind of cost function, taking into account, for example, total assembly time and used resources. This optimal sequence can be generated using some Branch-and-Bound method [33]. Because of the combinatorial explosion, there are many sequences to investigate, and heuristic search methods are used for faster retrieving the optimal sequence.

Because of the need for specific resources during flexible assembly - such as grippers, feeders and fixtures - it is not very useful to determine the optimal sequence off line, and to store it together with the product model. During actual assembly, the availability of the resources can vary. One reason for this is the unpredictable time a resource is actually used during assembly, because of all kinds of uncertainties that may arise during assembly. It is therefore better to calculate the optimal sequence on line, just before the actual assembly takes place, so that the available resources can be taken into account, and uncertainties have been eliminated as much as possible [34].

\subsubsection{Additional profits of using features for sequence planning}

Usually, the selection of the next candidate component to be evaluated for disassembly is done by a time-consuming trial-and-error method. One by one, every component in the partial assembly left is chosen, and is evaluated to see whether it can be disassembled.

More sophisticated knowledge can be used, because some features already contain information about possible assembly sequences. By using connection feature information, a reduction in search space can be realized. Connection features can often give information on the precedences of the components involved in the connection, resulting in a priority for the selection of the next component to evaluate.

Connection features with agents, can quickly give the component to select first, because they 'know' something about the disassembly sequence. It is useless to try to disassemble a plate when it is still connected by a bolt, so the connection feature will present the bolt first, and after that the plate connected by the bolt. Take, for example, the product of Fig. 22. In Fig. 23(a), the search space is shown when no feature information is used. Between the base and the plate there exists a specific attachment, say a pattern of threaded connections. This specific attach-

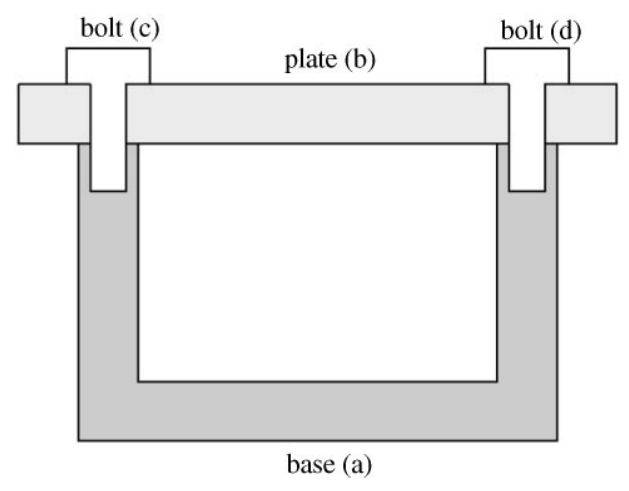

Fig. 22. Simple product.

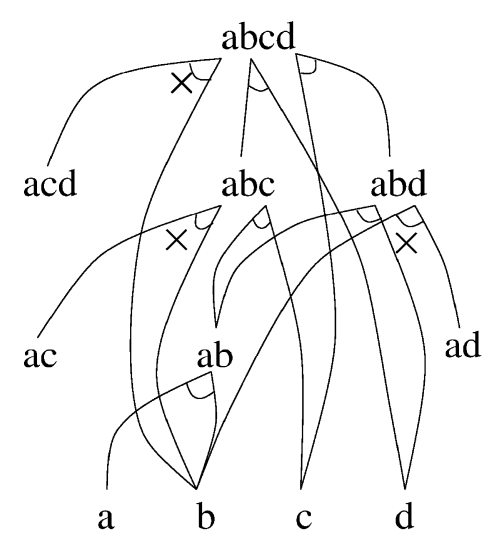

(a)

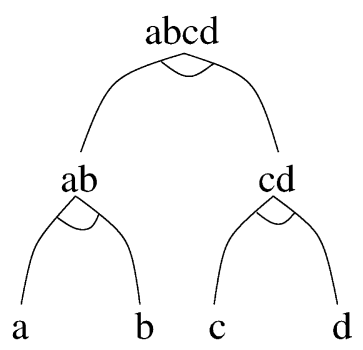

(b)

Fig. 23. AND/OR graphs of product shown in Fig. 22, without (a) and with (b) using feature information. Note that the AND relations in (a) marked with a cross represent search directions initially followed but giving a negative result, because the represented (dis)assembly operation cannot be performed.

ment contains two bolts as component agents, and two attachments as connection agents. These attachments are the threaded connections, each of them containing one bolt as the component agent, and a threaded pen-hole and a normal pen-hole connection as connection agents.

A connection feature representing a pattern can directly indicate that it does not matter in which order to disassemble the components, e.g. that it does not matter in which order a pattern of bolts is assembled. In the 
example above, this means that the search space is reduced because it does not matter whether first bolt $\mathrm{c}$ is assembled, or bolt $\mathrm{d}$. The attachments, i.e. the threaded connections, further reduce the search space, because these attachments directly indicate the assembly order for base, plate and bolt: first the base, then the plate, and finally the bolt. There is no need to search for alternative solutions here. Fig. 23(b) shows the reduced search space when feature information is taken into account.

Thus, by using feature information, the AND/OR graph can shrink significantly, resulting in a smaller number of sequences that have to be checked. This will result in much lower computation times for the assembly sequence planner.

\section{Conclusions}

The concept of combining a single-part feature model and an assembly feature model, has resulted in a very powerful integrated object-oriented product model. Modeling of single parts and of assemblies, is in this concept based on the same structure, providing a uniform way of working. Therefore an important step to integrate single-part and assembly modeling in one system has been made.

Just as features are a valuable concept in manufacturing, they are also useful in assembly. Assembly features, i.e. handling and connection features, are modeling entities on a conceptually higher level than those provided by geometric modelers. More assembly information known during design can be stored, and be used in subsequent phases of the product life cycle.

Connection features can be a basis for a relationdriven modeling concept. Within this concept, a model starts with raw geometry, and the relations between the geometric elements create more details of the shapes. This concept can be useful in bridging the current gap between functional and geometric modelers.

Assembly features can be profitably used in assembly planning modules. This has been shown for stability analysis, grip planning, motion planning and assembly sequence planning, but is likely also the case for other assembly planning modules. The modules cannot only be used in the actual assembly planning phase, but also in the modeling phase to check whether certain requirements related to assembly are fulfilled, which can be used to implement the DFA concept.

Therefore, the feature concept does not only integrate single-part and assembly modeling, but also modeling and planning. With the implementation of the prototype system, it has been demonstrated that both integrations are feasible (see [35]) for more details). Further development of the system can therefore result in a powerful integrated modeling and planning system for complex products.

\section{References}

[1] Boothroyd G, Dewhurst P, Knight W. Product design for manufacture and assembly. NY, USA: Marcel Dekker Inc, 1994.

[2] Shah JJ, Mäntylä M. Parametric and feature-based CAD/CAM. New York, USA: Wiley, 1995.

[3] Shah JJ, Rogers MT. Assembly modeling as an extension of feature-based design. Research in Engineering Design 1993;5(3\&4): 218-37.

[4] Rumbaugh J, Blaha M, Premerlani W, Eddy F, Lorensen W. Object-oriented modeling and design. Englewood Cliffs, New Jersey, USA: Prentice-Hall International Inc, 1991.

[5] Ovtcharova J, Pahl G, Rix J. A proposal for feature classification in feature-based design. Comput Graphics 1992;16(2): 187-95.

[6] Dohmen M. A survey of constraint satisfaction techniques for geometric modeling. Comput Graphics 1995;19(6):831-45.

[7] Lee K, Andrews G. Inference of positions of components in an assembly: part 2. Comput-Aided Des 1985;17(1):20-4.

[8] De Fazio TL. A prototype of feature-based design for assembly. In: Ravani B, editor. ASME Advances in Design Automation 1990, Chicago, IL, USA, 1990. p. 9-16.

[9] Sodhi R, Turner JU. Representing tolerance and assembly information in a feature-based design environment. In: Gabriele GA, editor. Proceedings of the 1991 ASME Design Automation Conference, vol. DE-vol. 32-1, Miami, Florida, USA, 1991. p. $101-8$.

[10] Shah JJ, Tadepalli R. Feature based assembly modeling. In: Gabriele GA, editor. Proceedings of the 1992 ASME International Computers in Engineering Conference, vol. 1, San Francisco, California, USA, 1992. p. 253-60.

[11] Sanderson AC, Homem de Mello LS, Zhang H. Assembly sequence planning. AI Mag Spring 1990:62-80.

[12] Zink R. XHDG (X toolkit Hierarchical Directed Graphs). URL: http://www.informatik.uni-stuttgart.de/ipvr/as/projekte/ grids/xhdg/xhdg-e.html, 1994.

[13] Homem de Mello LS, Sanderson AC. A basic algorithm for the generation of mechanical assembly sequences. In: Homem de Mello LS, Lee S, editors. Computer-aided mechanical assembly planning. Dordrecht, The Netherlands: Kluwer Academic Publishers, 1991. p. 163-90.

[14] Wolter JD. On the automatic generation of assembly plans. In: Homem de Mello LS, Lee S, editors. Computer-aided mechanical assembly planning. Dordrecht, The Netherlands: Kluwer Academic Publishers, 1991. p. 263-88.

[15] Delchambre A. Computer-aided assembly planning. London, UK: Chapman \& Hall, 1992.

[16] Nevins J, Whitney DE, editors. Concurrent design of products and processes. New York, USA: McGraw-Hill, 1989.

[17] Gottschlich S, Ramos C, Lyons D. Assembly and task planning: a taxonomy. IEEE Robotics Automat Mag 1994;1(3):4-12.

[18] Boneschanscher N. Plan generation for flexible assembly systems. PhD thesis, Delft University of Technology, The Netherlands, 1993.

[19] Lee S, Yi C. Subassembly stability and reorientation. Proceedings of the IEEE International Conference on Robotics and Automation, vol. 2, Atlanta, GA, USA, 1993. p. 521-6.

[20] Chen L-L, Chou S-Y, Woo TC. Parting directions for mould and die design. Comput-Aided Des 1993;25(12):762-8.

[21] Chen L-L, Chou S-Y, Woo TC. Separating and intersecting spherical polygons: computing machinability on three-, four-, and five-axis numerically controlled machines. ACM Trans Graphics 1993;12(4):305-26.

[22] Mattikalli RS, Khosla PK. Motion constraints from contact geometry: representation and analysis. Proceedings of the IEEE International Conference on Robotics \& Automation, Nice, France, 1992. p. 2178-85. 
[23] Mattikalli R, Baraff D, Khosla P. Finding all gravitationally stable orientations of assemblies. Proceedings of the IEEE International Conference on Robotics and Automation, vol. 1, San Diego, CA, USA, 1994. p. 251-7.

[24] van Holland W, Bronsvoort WF. Assembly features and visibility maps. In: Busnaina AA. editor. Proceedings of the 15th ASME International Computers in Engineering Conference, Boston, MA, USA, 1995. p. 691-7.

[25] Mattikalli RS, Khosla PK. Analysis of restraints to translational and rotational motion from the geometry of contact. In: Sharon, A, Behun R, Prinz F, Young L, editors. Issues in design manufacture/integration, Winter Annual Meeting of ASME, vol. 39, Atlanta, GA, USA, 1991. p. 65-71.

[26] van Bruggen M, Baartman JP, Bronsvoort WF. Grips on parts. Proceedings of the IEEE International Conference on Robotics and Automation, vol. 2, Atlanta, GA, USA, 1993. p. 828-33.

[27] Baartman JP. Automation of assembly operations on parts. $\mathrm{PhD}$ thesis, Delft University of Technology, The Netherlands, 1995.

[28] van Holland W, Bronsvoort WF. Extracting grip areas from feature information. In: McCarthy JM, editor. Proceedings of the ASME 1996 Design Engineering Technical Conferences and Computers in Engineering Conference, Irvine, CA, USA, 1996. Published on cdrom, ISBN 0-7918-1232-4.
[29] De Fazio TL, Whitney DE. Simplified generation of all mechanical assembly sequences. IEEE J Robot Automat 1987;RA3(6):640-58.

[30] Lozano-Pèrez T, Wilson RH. Assembly sequencing for arbitrary motions. Proceedings of the IEEE International Conference on Robotics and Automation, Atlanta, GA, USA, 1993. p. 527-32.

[31] Chakrabarty S, Wolter J. A hierarchical approach to assembly planning. Proceedings of the IEEE International Conference on Robotics and Automation, vol. 1, San Diego, CA, USA, 1994. p. 258-63.

[32] Homem de Mello LS, Sanderson AC. Representations for assembly sequences. In: Homem de Mello LS, Lee S, editors. Computer-aided mechanical assembly planning. Dordrecht, The Netherlands: Kluwer Academic Publishers, 1991. p. 129-62.

[33] Barr A, Feigenbaum EA. The handbook of artificial intelligence, vol. 1. Los Altos, CA, USA: Stanford Heuristic Press and William Kaufmann Inc, 1981.

[34] van Holland W, Boneschanscher N, Bronsvoort WF. Task assignment in a flexible assembly cell using and/or graphs. In: Basañez L, editor. International Symposium on Industrial Robots, Barcelona, Spain, 1992. p. $653-8+642$.

[35] van Holland W. Assembly features in modeling and planning. PhD thesis, Delft University of Technology, The Netherlands, 1997. 